\title{
CALCULATIONS OF SURFACE ROUGHNESS 3D PARAMETERS FOR SURFACES WITH IRREGULAR ROUGHNESS
}

\author{
Natalija Bulaha \\ Riga Technical University, Latvia \\ natalija.bulaha@rtu.lv
}

\begin{abstract}
In this scientific work, an analysis of surface roughness measurements was performed with the aim of determining the correlations (equations) for calculating 3D roughness parameters. The obtained formulas will enable to predict the values of roughness parameters and make conclusions about the surface exploitational characteristics. In the given work, surfaces with irregular roughness were investigated, i.e. flat grinded, cylindrically grinded, polished, lapped etc, using the modern measuring equipment Taylor Hobson Talysurf Intra 50 and the data processing software $\mu$ ultra. The second and most important task of this work was the analytical calculations of $3 \mathrm{D}$ roughness parameters using the random field theory and its basic principles. The calculations were performed on the parameters $S a, S p, S d r, S d q, S p c, V m, S m r$, which are standardized in ISO 25178-2:2012. After the calculations, the results were compared with the measurement data. The differences in the values of the roughness parameters were $\leq 10 \%$, but in some cases the error exceeded $10 \%$, which could be explained by possible surface defects or insufficient measuring area.
\end{abstract}

Keywords: surface, roughness parameters, random field, wear, adhesion.

\section{Introduction}

Nowadays, the quality of produced components, units and constructions influence effective operation and service life. In the field of mechanical engineering, technologists and constructors often encounter such phenomena as surface wear, contact deformations, adhesion, reflection of light, etc. It is important to determine and control the surface roughness parameters to ensure the required properties of the surface.

Technologically, it is possible to ensure only a few roughness parameters during the components' production: the mean arithmetic height $\mathrm{Sa}[1]$ and the mean width of the roughness profile elements $R S m$ [2], varying with cutting regimes such as feed, cutting speed and depth. The given basic parameters make it possible to predict values of other 3D parameters (material volume, texture aspect ratio, arithmetic mean peak curvature, root mean square gradient, etc.), depending on the type of the surface roughness.

As for surface roughness irregularities, it is important to determine their type, because the calculations of 3D parameters are based on the random field theory, which is valid only for surfaces with irregular roughness, when surface irregularities are placed chaotically and have irregular form. This type of surface can be obtained by grinding, polishing and other types of processing, where abrasive material (grains) serves as a cutting tool.

It is important to make experiments with several sample batches to get reliable data. In the given work seven types of surfaces with irregular roughness were examined, with the aim of determining the equations for 3D roughness parameter calculation.

\section{Materials and methods}

The first step in this work was performance of the roughness measuring experiments. The modern equipment Taylor Hobson Talysurf Intra 50 (Fig. 1) was selected for fulfilment of the task. For the given equipment, the measurement process is carried out with a diamond stylus that moves over the sample's surface. The given equipment has a $16 \mathrm{~nm}$ resolution, radius of needle and standard stylus arm 112/2009.

Data processing was carried out with the help of the computer program $\mu$ ultra. Each surface was subjected to levelling, form and waviness removal with the aim of obtaining the surface roughness topography and roughness parameters.

Several series of samples were selected for carrying out the experiments:

1. flat grinding - Rugotest 104 - Surface No.2, 7, 8;

2. cylindrical grinding - Rugotest 105 - Surface No.4, 5, 7 (see Fig. 2);

3. lapping - Rugotest - Surface No.1, 2, 3; 
4. polishing - Rugotest - Surface No.1, 2, 3;

5. shot peening - Rugotest 3B - Surface No.6, 7, 8;

6. electroerosion - Rugotest 107 - Surface No.6, 7, 8;

7. sand blasting - Rugotest $3 \mathrm{~A}-$ Surface No.6, 7,8 .

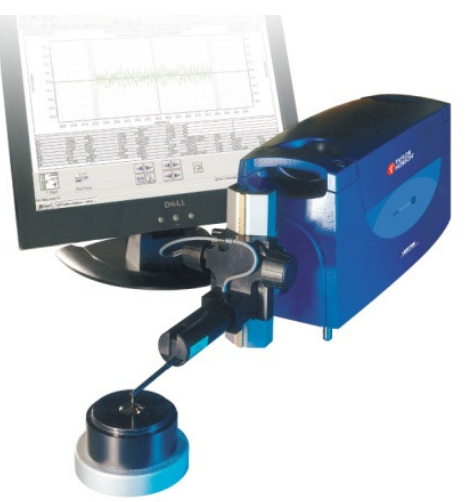

Fig. 1. Measuring equipment Taylor Hobson Talysurf Intra 50

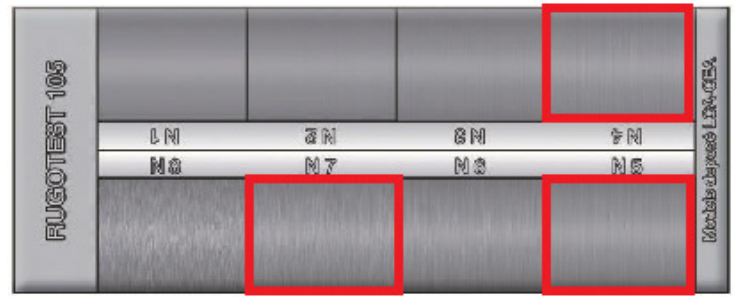

Fig. 2. Sample Rugotest 105 - Cylindrical grinding

There were measured three surfaces with different values of the parameter Sa for each series of samples.

The next step was to analyse the calculation formula for the 3D roughness parameters, based on the random field theory. The random field theory fits for surfaces with irregular roughness. Theoretically and practically, it has been proven that such types of surfaces are characterized by normal Gaussian distribution of ordinates, skewness $S s k=0$ and kurtosis $S k u=3$ [3]. The correlation functions for such surfaces are monotonically decreasing and/or decreasing with oscillations, depending on the average steps of roughness microirregularities in the $\mathrm{X}$ and $\mathrm{Y}$ directions.

The parameter $S a$ - arithmetical mean height, which characterizes the average amplitude of surface roughness. The values of this parameter are closely related to the root mean square height $S q$ and are calculated by the following formula [4]:

$$
E\{S a\} \approx S q \cdot \sqrt{\frac{2}{\pi}}
$$

where $S a$-arithmetical mean height, $\mu \mathrm{m}$;

$S q$ - root mean square height, $\mu \mathrm{m}$.

The calculated values of the roughness parameter Sa for all surfaces correspond to Taylor Hobson's data and fall within a range of possible deviations of $\pm 10 \%$. This can be justified by Fig. 3 . data.

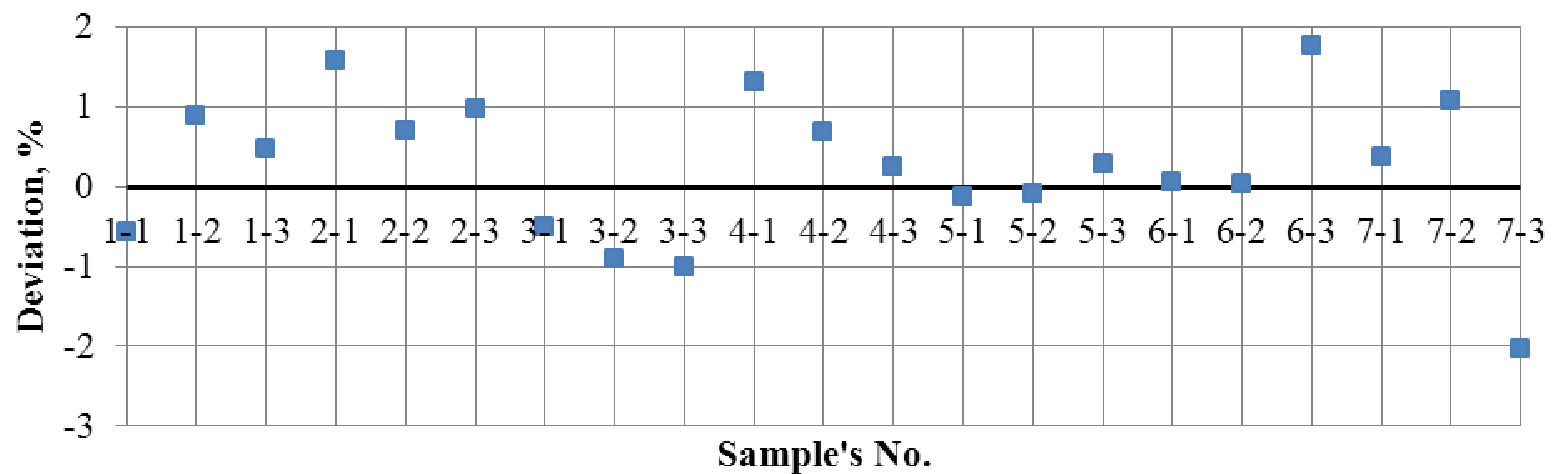

Fig. 3. Deviations of calculated and measured values of parameter Sa

Generally, it is difficult to estimate the shape and location of surface roughness by the parameter $\mathrm{Sa}$, but knowing the values of kurtosis $S k u$ and skewness $S s k$ it can be predicted. As mentioned above, 
for surfaces with $S k u=3$ and $S s k=0$, surface ordinates' distribution will abide the normal Gauss distribution law (Fig. 4).

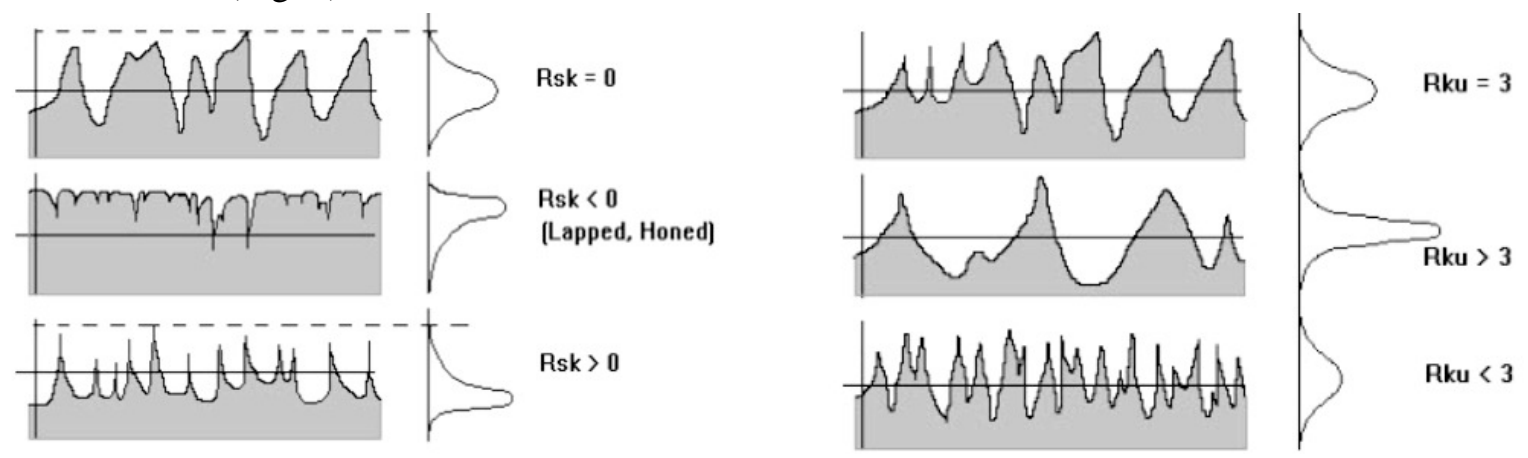

Fig. 4. Depiction of roughness parameters Ssk and Sku [5]

The next parameter is the maximum peak height $S p$; it is a distance between the mean plane of the surface and the highest peak. This parameter is important for estimating the components' contact, identification of individual irregularities, burrs or other defects. The given parameter is expressed by formula [4]:

$$
E\{S p\} \approx 2 \cdot S q \cdot \sqrt{\ln \left[E\left\{N_{01}\right\} \cdot \sqrt{S t r}\right]}
$$

where $S p$ - maximum peak height, $\mu$ m;

$E\left\{N_{01}\right\}$ - number of intersections with a mean line;

Str - texture aspect ratio

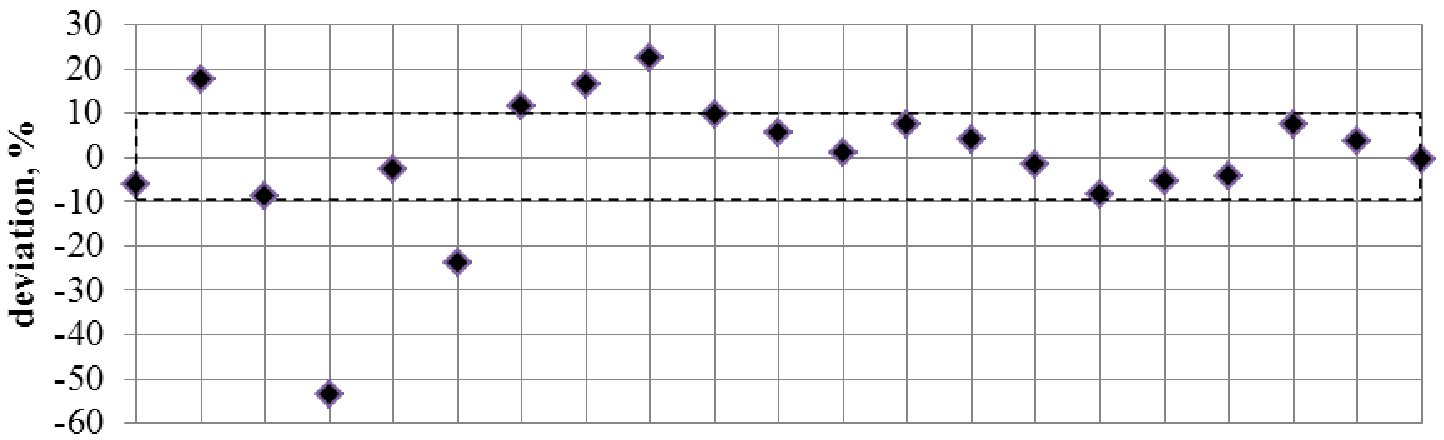

1-1 1-2 1-3 2-1 2-2 2-3 3-1 3-2 3-3 4-1 4-2 $4-3$ 5-1 5-2 5-3 6-1 6-2 6-3 7-1 7-2 7-3

Sample's No.

Fig. 5. Deviations of calculated and measured values of parameter $S p$

According to Fig. 5, it can be concluded that the calculated $S p$ values for the most part of the samples fit into the range $\pm 10 \%$ of Taylor Hobson values; so it can be concluded that the equation (2) can be used to determine the given parameter.

The next parameter is the arithmetic mean peak curvature $S p c$, which determines the mean radius of the surface microirregularities, what is very important for the analysis of friction surfaces. This parameter influences wear rate of the surface. It was justified that the roughness irregularities with smaller radius of peaks will have less contact area, while contacting with solid smooth surface [6;7]. Depending on the values of the parameter $S p c$, there will be changes in the surface deformation type: plastic, elastic or elastic-plastic. The given parameter is calculated according to the following formula [4]:

$$
E\{S p c\} \approx \frac{1}{2} \cdot \pi^{2} \cdot S q \cdot\left(\frac{2}{R S m_{1}}\right)^{2} \cdot\left(1+S t r^{2}\right) \gamma,
$$

where $R S m_{1}$ - mean width of the roughness profile elements (taken from three profilograms), $\mathrm{mm}$; $\gamma$ - level from the mean line. 
The equation (3) expresses the parameter $S p c$ at different levels, for example, $\gamma=2,2.5,3$ from the mean plane. The values of this parameter for all three levels are shown in Fig. 6:

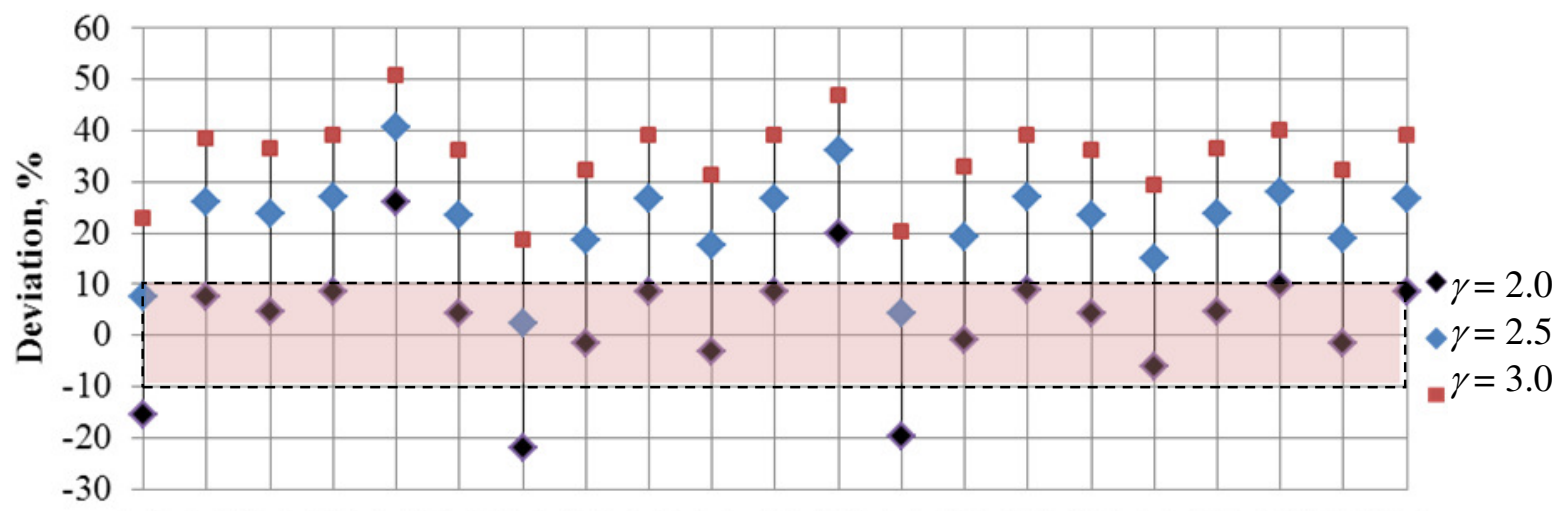

1-1 1-2 1-3 2-1 2-2 2-3 3-1 3-2 3-3 4-1 4-2 4-3 5-1 5-2 5-3 6-1 6-2 6-3 7-1 7-2 7-3

Sample's No.

Fig. 6. Deviations of calculated and measured values of parameter Spc

After the analysis of Fig. 6, it can be concluded that the calculated values of the parameter Spc correspond to measured at the level $\gamma=2$. At the higher levels, Spc calculated values do not fall into the interval of deviations $\pm 10 \%$.

When studying the surface aerodynamic properties and hydrodynamic resistance, it is necessary to take into account the root mean square gradient $S d q$. After the theoretical-experimental conclusions [8] the relationship between the tractive force and the angle of roughness irregularities for calculation of aerodynamic parameters was determined, as well as the relationship between the roughness tangent angle and the hydraulic resistance for determination of the roughness influence on resistance to motion. The Sdq parameter can be calculated using the formula [4]:

$$
E\{S d q\} \approx \frac{\pi}{2} \cdot S q \cdot \frac{2}{R S m_{1}} \cdot \sqrt{\pi \cdot\left(1+S t r^{2}\right)}
$$

The comparison of the calculated and measured values of this parameter is shown in Fig. 7.

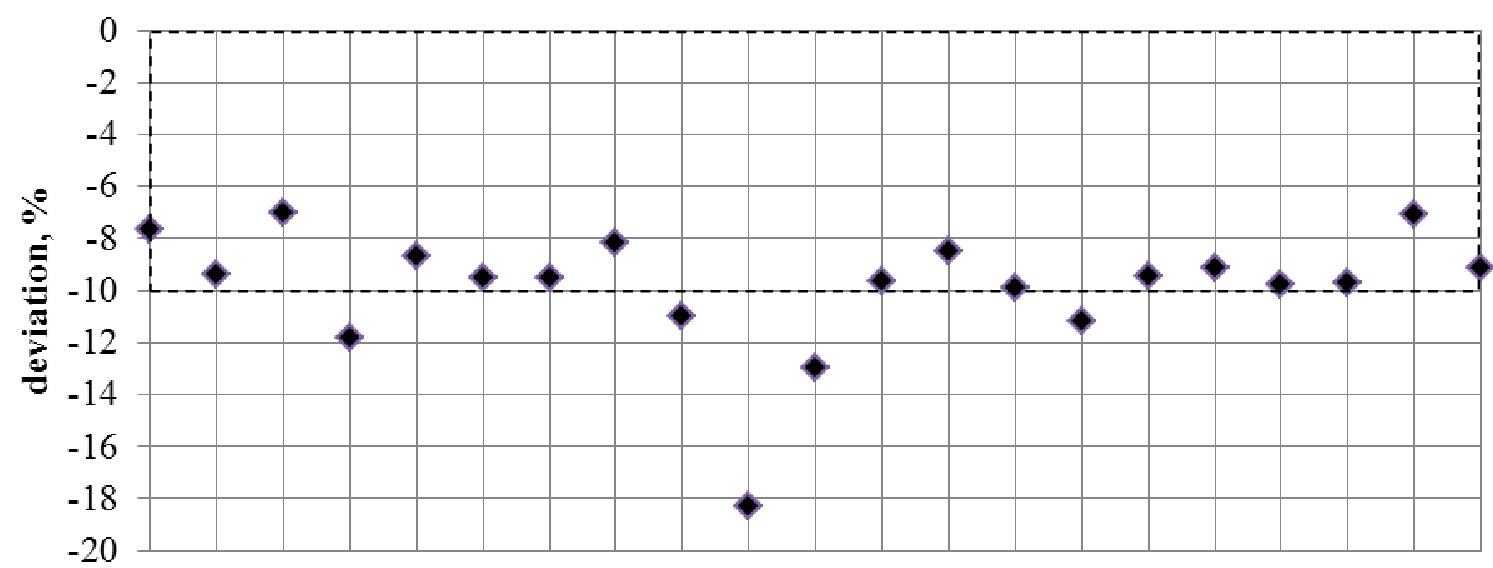

$\begin{array}{lllllllllllllllllllll}1-1 & 1-2 & 1-3 & 2-1 & 2-2 & 2-3 & 3-1 & 3-2 & 3-3 & 4-1 & 4-2 & 4-3 & 5-1 & 5-2 & 5-3 & 6-1 & 6-2 & 6-3 & 7-1 & 7-2 & 7-3\end{array}$

Sample's No.

Fig. 7. Deviations of calculated and measured values of parameter $S d q$

According to the obtained data, it is seen that almost all of errors of the parameter $S d q$ are in the deviation interval $\pm 10 \%$, which implies that the given equation can be used in determination of the root mean square gradient. 
The parameter $S d r$ - developed interfacial area ratio is important for surface adhesion issues. How well the coating will remain on the surface depends on the number of molecular links between the base material and the adhesive, what, in turn, is determined by the ratio between the actual and nominal surface area. In this case, the values of this parameter will depend on the average arithmetic height $S a$, the large values of which will increase the actual surface area [9]. The parameter $S d r$ is calculated by the formula [4].

$$
E\{S d r\} \approx \frac{\pi^{2}}{2} \cdot S q^{2} \cdot\left(\frac{2}{R S m_{1}}\right)^{2} \cdot\left(1+S t r^{2}\right) \cdot 100 \%
$$

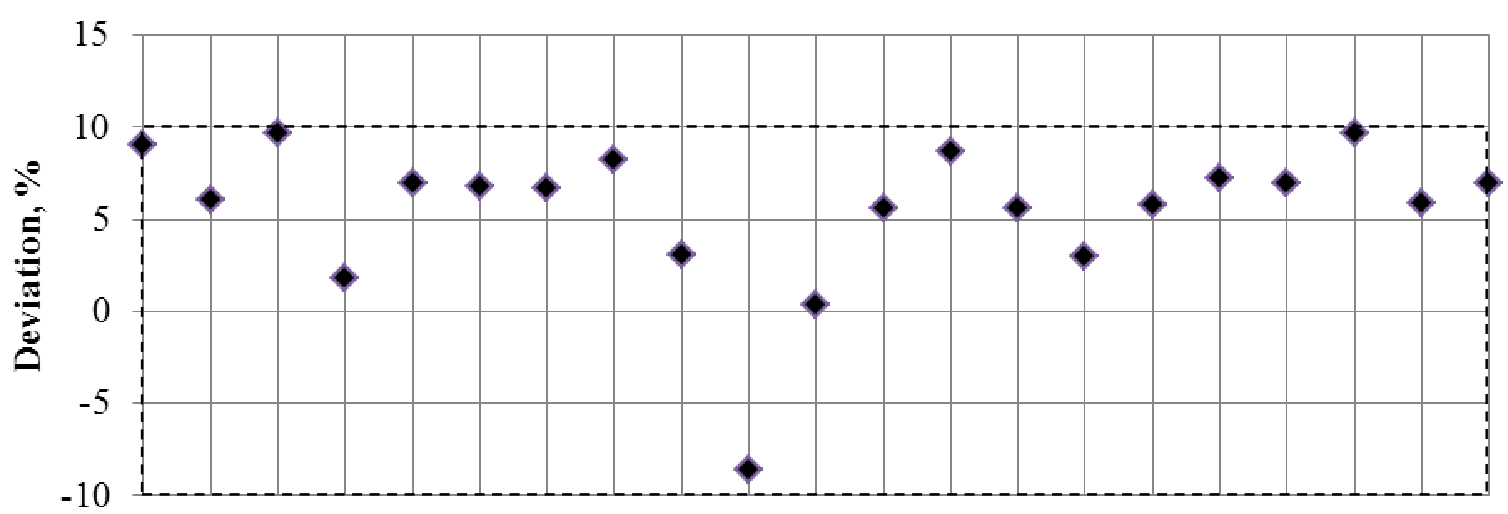

$\begin{array}{lllllllllllllllllllll}1-1 & 1-2 & 1-3 & 2-1 & 2-2 & 2-3 & 3-1 & 3-2 & 3-3 & 4-1 & 4-2 & 4-3 & 5-1 & 5-2 & 5-3 & 6-1 & 6-2 & 6-3 & 7-1 & 7-2 & 7-3\end{array}$

Sample's No.

Fig. 8. Deviations of calculated and measured values of parameter $S d r$

Fig. 8 shows that there is a very small difference between the measured and calculated values of the parameter $S d r$, which is smaller than $10 \%$, so it can be concluded that the equation (5) can be used for determination of the developed interfacial area ratio.

Another important parameter is the material volume $\mathrm{Vm}$, which is determined at different levels from the deepest valley. Taylor Hobson software also gives values for this parameter, depending on the level, but deducting from the highest peak.

The given parameter is calculated according to the following formula [4]:

$$
E\left\{V_{u}\right\}=S q \cdot A\left\{\frac{1}{\sqrt{2 \pi}} \exp \left(-\frac{u^{2}}{2 S q^{2}}\right)-\frac{u}{S q}\left[1-\phi\left(\frac{u}{S q}\right)\right]\right\} .
$$

where

$$
u=\gamma \cdot S q
$$

In the given work the calculations of the parameter $\mathrm{Vm}$ were made only at one level - from the deepest peak to the highest point of the surface - at the level $u=-3 S q-0.5 x$, because, assuming that $99.7 \%$ of all ordinate values are in the range of $6 S q$, the remaining part will be $x$ (see Table 1, Fig. 9).

\section{Summary of parameters}

\begin{tabular}{|c|c|c|}
\hline Parameter & Value & Notes \\
\hline$S t / S q$ & 7.30 & $>6 S q$ \\
\hline $6 S q$ & $10.68 \mu \mathrm{m}$ & Interval for $99.7 \%$ of all ordinates' values \\
\hline $0.5 x=(S t-6 S q) / 2$ & $1.16 \mu \mathrm{m}$ & Remaining part \\
\hline$u_{\max }=3 S q+0.5 x$ & $6.5 \mu \mathrm{m}$ & Maximal level u \\
\hline
\end{tabular}




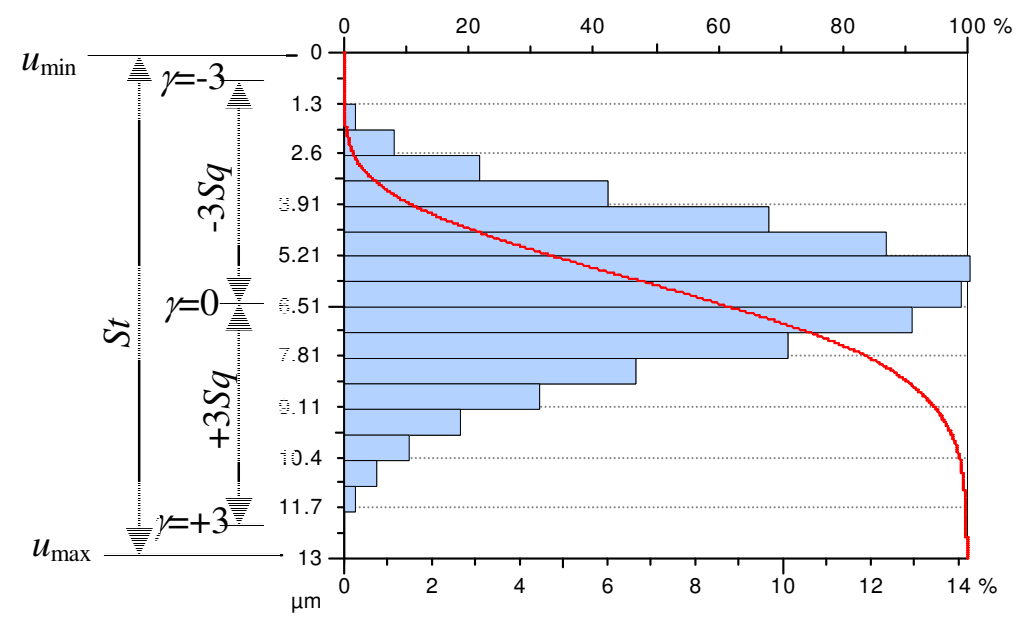

Fig. 9. Ordinates' distribution

The results given in Fig. 10 show that a large number of deviations for calculated and measured values of the parameter $\mathrm{Vm}$ are in the range of $\pm 10 \%$.

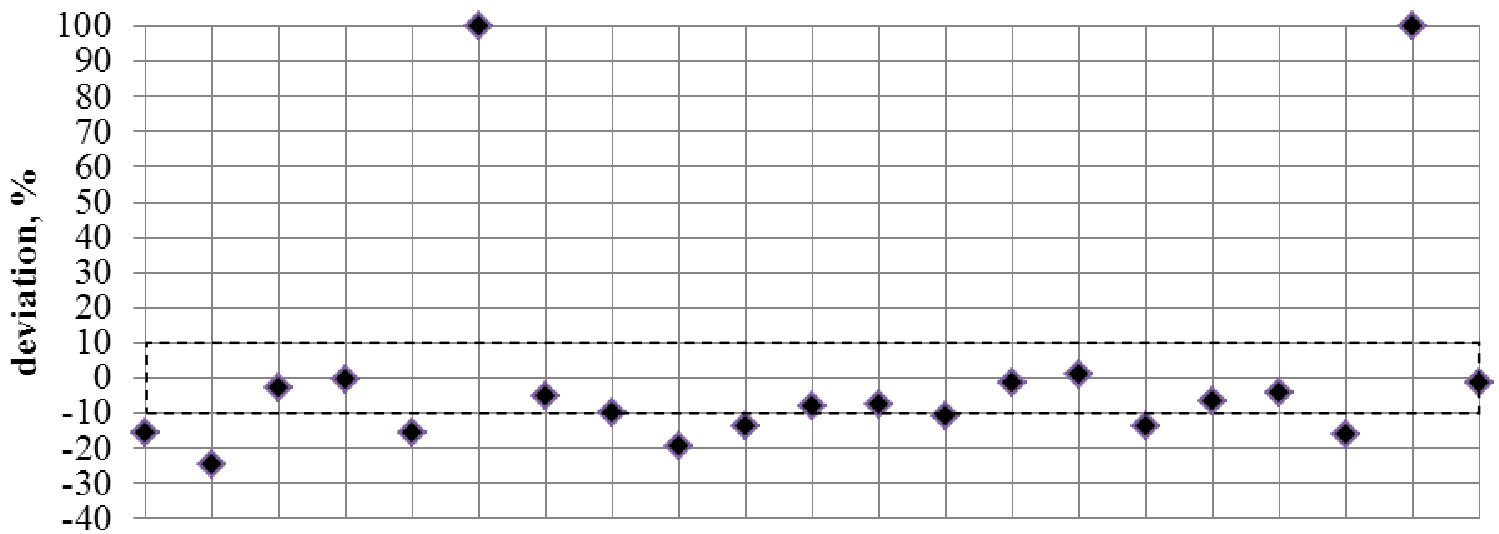

$\begin{array}{lllllllllllllllllllll}1-1 & 1-2 & 1-3 & 2-1 & 2-2 & 2-3 & 3-1 & 3-2 & 3-3 & 4-1 & 4-2 & 4-3 & 5-1 & 5-2 & 5-3 & 6-1 & 6-2 & 6-3 & 7-1 & 7-2 & 7-3\end{array}$ Sample's No.

Fig. 10. Deviations of calculated and measured values of parameter $\mathrm{Vm}$

Areal material ratio Smr is the ratio between the sum of intersection areas of microirregularities at the level $u$ and the nominal area. As mentioned above, the standard levels are: $\gamma=0 ; 1 ; 2 ; 3 ;-1 ;-2 ;-3$. Each surface will have a different value of the parameter Smr and, accordingly - the level $\gamma$. For surface with normal Gaussian distribution of ordinates, the $\mathrm{Smr}$ values at different levels will correspond to integral Laplace distribution function. The theoretical values of Smr are calculated using the following formula [4]:

$$
E\left\{\eta_{u}\right\}=1-\Phi\left(\frac{u}{\sigma}\right) .
$$

The $S m r$ values of a real surface are given by the Taylor Hobson program $\mu$ ultra, where there is a possibility to select the required level by calculating it beforehand.

The given parameter gives an opportunity to determine whether the ordinates of the surface are distributed by the normal Gaussian distribution law, in addition to determining of $S k u, S s k$ and the Pearson criterion. By itself this parameter describes how large the supporting area is at different stages of wear.

A comparison of the real and theoretical Smr values is shown in Fig. 11. 


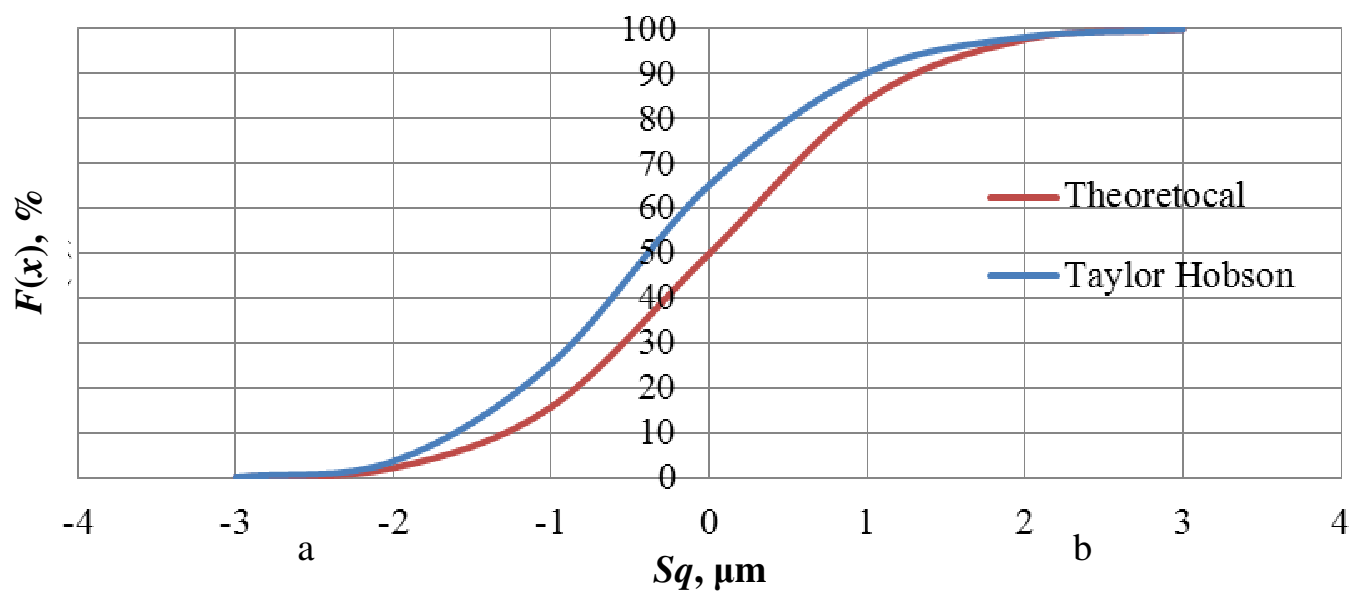

Fig. 11. Theoretical and real material ratio curves for flat grinded surface

According to Fig. 12 and 13 data, it is seen that the values of the parameter Smr mainly coincide with the results processed by Taylor Hobson only at the levels $\gamma=1,2,3$. At lower levels, the difference between the calculated and measured values is more than $\pm 10 \%$ and in some cases even reaches $100 \%$.

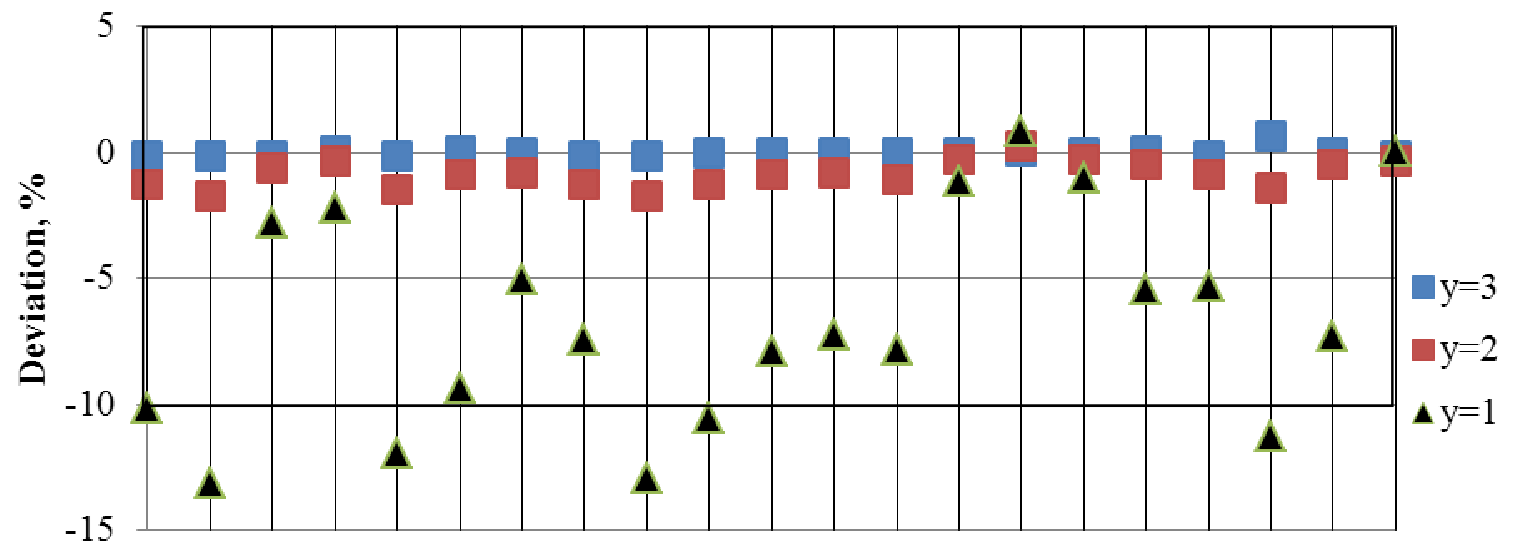

1-1 1-2 1-3 2-1 2-2 2-3 3-1 3-2 3-3 4-1 4-2 4-3 5-1 5-2 5-3 6-1 6-2 6-3 7-1 7-2 7-3

Sample's No.

Fig. 12. Deviations of calculated and measured values of parameter $\operatorname{Smr}$ at levels $\gamma=\mathbf{1 , 2 , 3}$

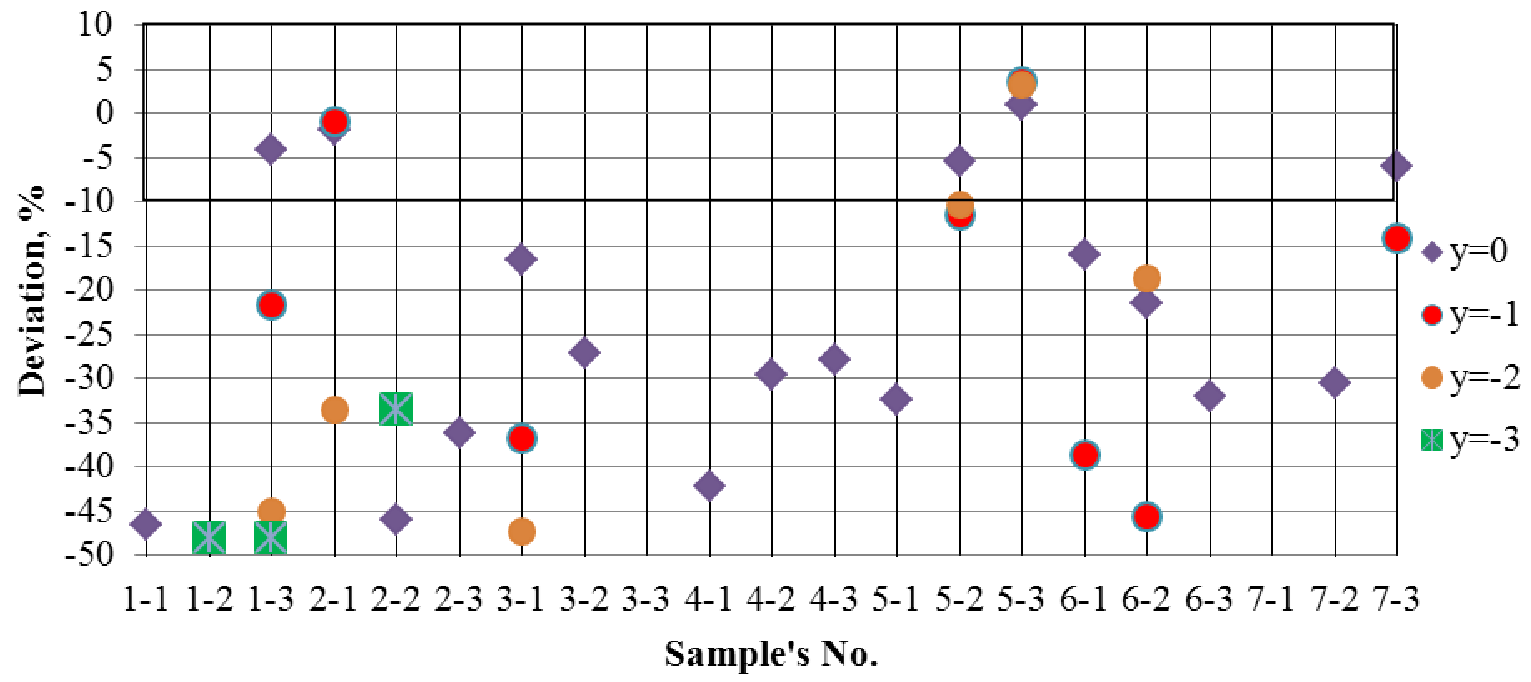

Fig. 13. Deviations of calculated and measured values of parameter $S m r$ at levels $\gamma=0,-1,-2,-3$ 


\section{Conclusions}

1. The formulas for 3D roughness parameter calculations are valid for surfaces with irregular roughness, which are characterized by normal Gaussian distribution of surface ordinates.

2. Comparison of the calculated and measured values of the parameters $S a, S p, S d q, S p c, S d r, V m$ and $S m r$ shows that the values vary in the range of $\pm 10 \%$, which allows us to assert that the calculation formulas considered in the given work can be used for determination and prediction of surface roughness parameters.

3. The deviations, which are larger than $\pm 10 \%$, can be explained by possible surface defects or insufficient measuring area.

4. The calculations of 3D roughness parameters are based on two parameters: $S a$-arithmetic mean height and $R S m$ - mean step of the roughness irregularities in the direction parallel and perpendicular to the processing traces.

\section{References}

[1] LVS EN ISO 25178-2:2012 standard "Geometrical product specifications (GPS) - Surface texture: Areal - Part 2: Terms, definitions and surface texture parameters"

[2] LVS EN ISO 4287:2002 standard "Geometrical Product Specifications (GPS) - Surface texture: Profile method - Terms, definitions and surface texture parameters"

[3] Bulaha N., Rudzitis J. Calculation possibilities of 3D parameters for surfaces with irregular roughness. Latvian Journal of Physics and Technical Sciences, Vol.3, 2018, 8 p. (unpublished)

[4] Рудзитис Я. Контактная механика поверхностей, 2ая часть - Микротопография шероховатости поверхности (Surface contact mechanics - part 2 - Surface roughness microtopography). Рига: РТУ, 2007. 213 стр. (In Russian).

[5] Laser Scanning on Road Pavements. [online] [14.03.2018.]. Available at: http://www.mdpi.com/1424-8220/12/7/9110/htm

[6] Donald K. Cohen. If you need less sliding friction, should you make the surface rougher or smoother? Quality magazine, 2017.

[7] Contact mechanics. [online] [15.03.2018.]. Available at: http://www.multiscaleconsulting.com/our-research/contact-mechanics

[8] Хусу А.П., Витенберг Ю.Р., Пальмов В.А. Шероховатость поверхностей: теоретиковероятностный подход (Surface roughness: theoretically-probabilistic approach). Москва: Наука, 1975. 344 стр. (In Russian).

[9] Michigan Metrology. [online] [14.02.2018.]. Available at: http://michmet.com/index.html 